Network Working Group

Request for Comments: 1658

Obsoletes: 1316

Category: Standards Track
B. Stewart

Xyplex, Inc. July 1994

\title{
Definitions of Managed Objects for Character Stream Devices using SMIv2
}

Status of this Memo

This document specifies an Internet standards track protocol for the Internet community, and requests discussion and suggestions for improvements. Please refer to the current edition of the "Internet Official Protocol Standards" (STD 1) for the standardization state and status of this protocol. Distribution of this memo is unlimited.

Table of Contents

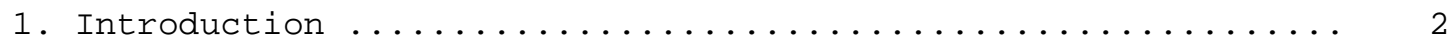

2. The SNMPv2 Network Management Framework ............... 2

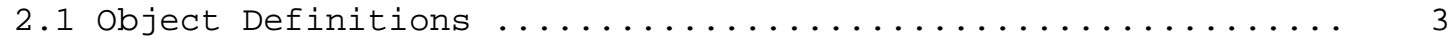

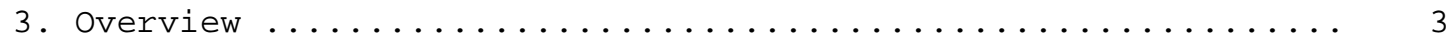

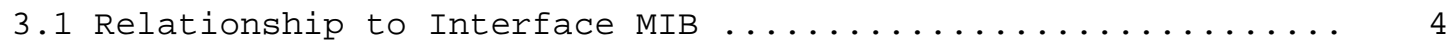

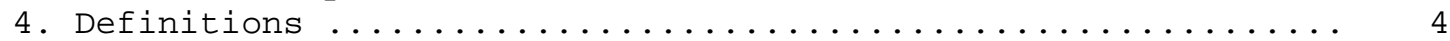

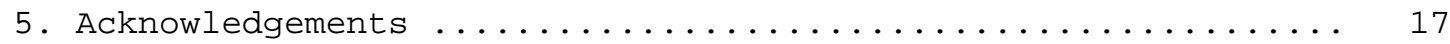

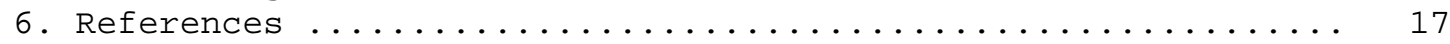

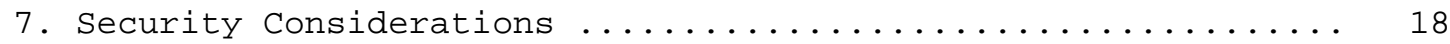

8. Author's Address .............................. 18

1. Introduction

This memo defines an extension to the Management Information Base (MIB) for use with network management protocols in the Internet community. In particular, it defines objects for the management of character stream devices.

2. The SNMPv2 Network Management Framework

The SNMPv2 Network Management Framework consists of four major components. They are:

o RFC 1442 [1] which defines the SMI, the mechanisms used for describing and naming objects for the purpose of management.

o STD 17, RFC 1213 [2] defines MIB-II, the core set of managed objects for the Internet suite of protocols. 
o RFC 1445 [3] which defines the administrative and other architectural aspects of the framework.

o RFC 1448 [4] which defines the protocol used for network access to managed objects.

The Framework permits new objects to be defined for the purpose of experimentation and evaluation.

\subsection{Object Definitions}

Managed objects are accessed via a virtual information store, termed the Management Information Base or MIB. Objects in the MIB are defined using the subset of Abstract Syntax Notation One (ASN.1) defined in the SMI. In particular, each object object type is named by an OBJECT IDENTIFIER, an administratively assigned name. The object type together with an object instance serves to uniquely identify a specific instantiation of the object. For human convenience, we often use a textual string, termed the descriptor, to refer to the object type.

\section{Overview}

The Character MIB applies to ports that carry a character stream, whether physical or virtual, serial or parallel, synchronous or asynchronous. The most common example of a character stream device is a hardware terminal port with an RS-232 interface. Another common hardware example is a parallel printer port, say with a Centronics interface. The concept also includes virtual terminal ports, such as a software connection point for a remote console.

The Character MIB is mandatory for all systems that offer character stream ports. This includes, for example, terminal servers, general-purpose time-sharing hosts, and even such systems as a bridge with a (virtual) console port. It may or may not include character ports that do not support network sessions, depending on the system's needs.

The Character MIB's central abstraction is a port. Physical ports have a one-to-one correspondence with hardware ports. Virtual ports are software entities analogous to physical ports, but with no hardware connector.

Each port supports one or more sessions. A session represents a virtual connection that carries characters between the port and some partner. Sessions typically operate over a stack of network protocols. A typical session, for example, uses Telnet over TCP. 
The MIB comprises one base object and two tables, detailed in the following sections. The tables contain objects for ports and sessions.

The MIB intentionally contains no distinction between what is often called permanent and operational or volatile data bases. For the purposes of this MIB, handling of such distinctions is implementation specific.

\subsection{Relationship to Interface MIB}

The Character MIB does not relate directly to the Interface MIB [1], since it is not intrinsically a network interface. On the other hand, in most implementations where it is present, it will be above a physical sublayer interface, such as the RS-232-like [2] or Parallel-printer-like [3] MIBs. Such physical interfaces typically are represented by a row in the interface table (ifTable), identified by a value of ifIndex.

4. Definitions

CHARACTER-MIB DEFINITIONS : := BEGIN

IMPORTS

MODULE-IDENTITY, OBJECT-TYPE, NOTIFICATION-TYPE,

Counter32, Integer32, Gauge32, TimeTicks FROM SNMPV2-SMI

Autonomoustype, InstancePointer FROM SNMPV2-TC

InterfaceIndex FROM IF-MIB

transmission, mib-2 FROM RFC1213-MIB

MODULE-COMPLIANCE, OBJECT-GROUP FROM SNMPV2-CONF ;

char MODULE-IDENTITY

LAST-UPDATED "9405261700Z"

ORGANIZATION "IETF Character MIB Working Group" CONTACT-INFO

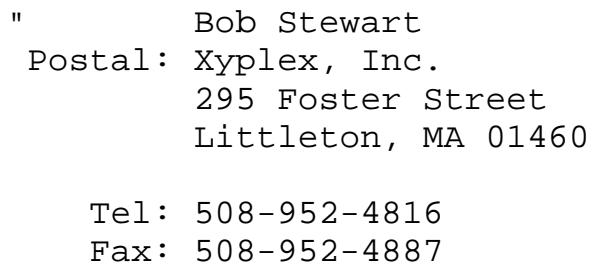

Tel: 508-952-4816

Fax: 508-952-4887 


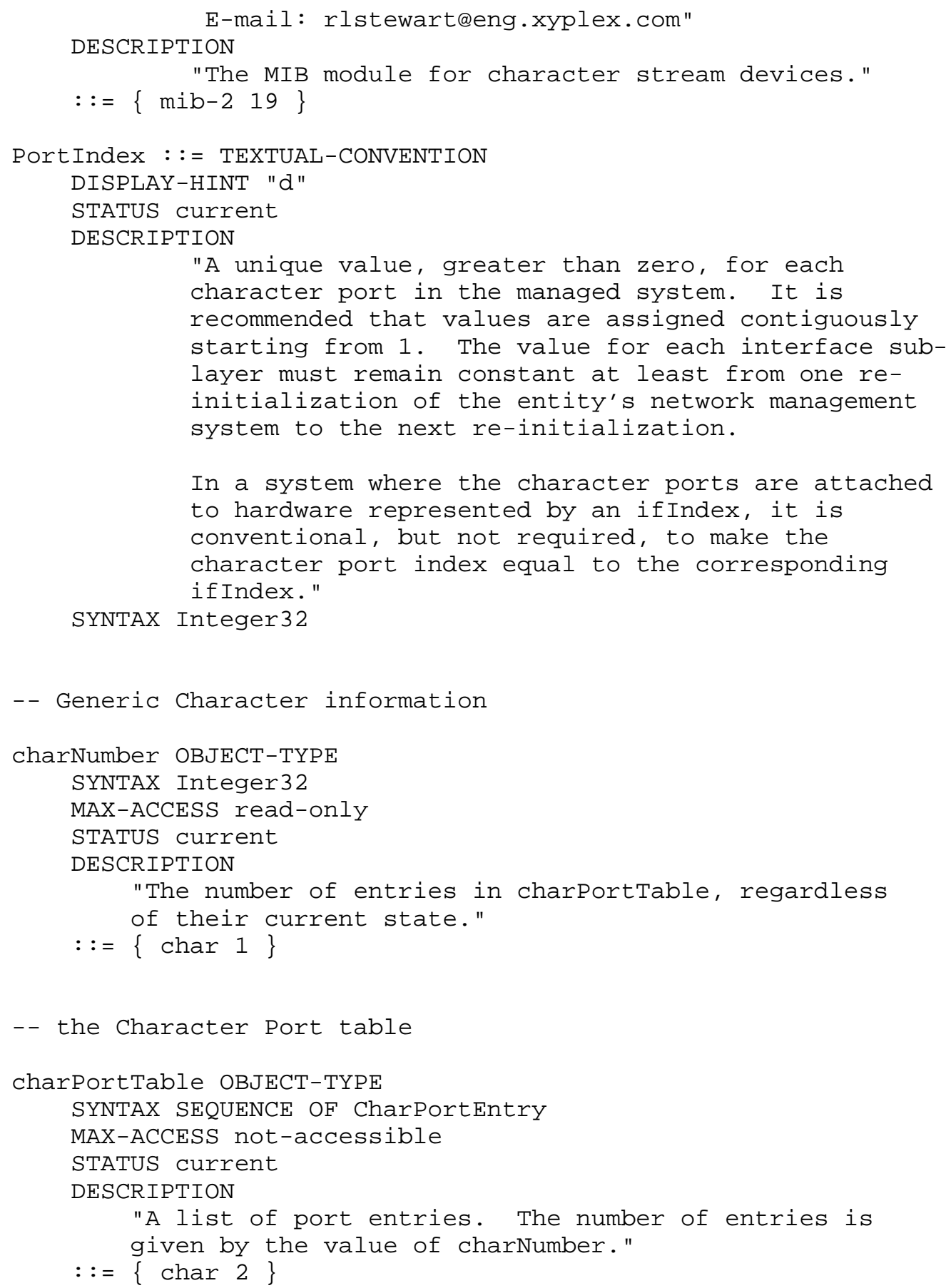




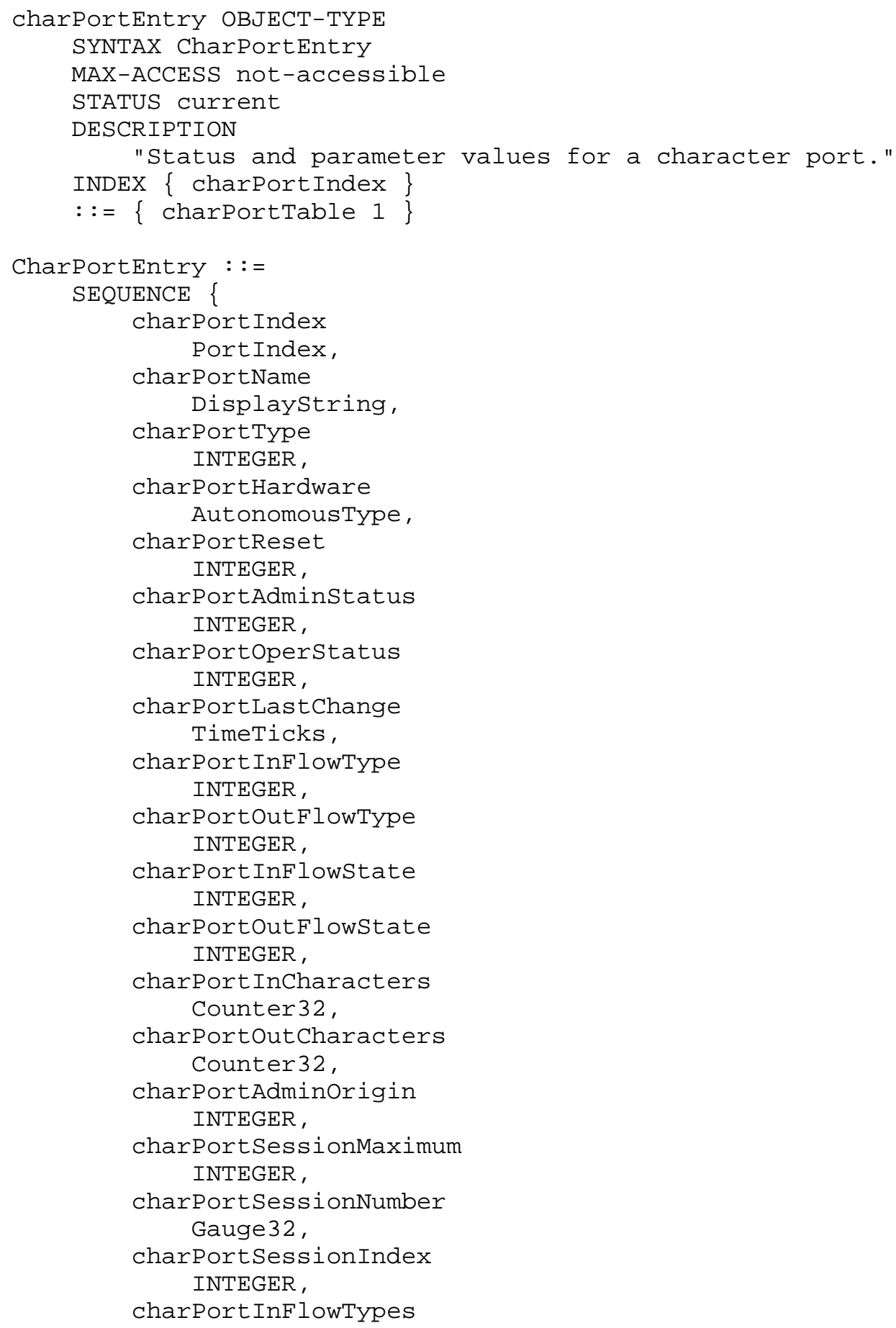




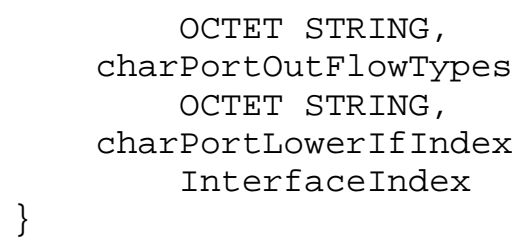




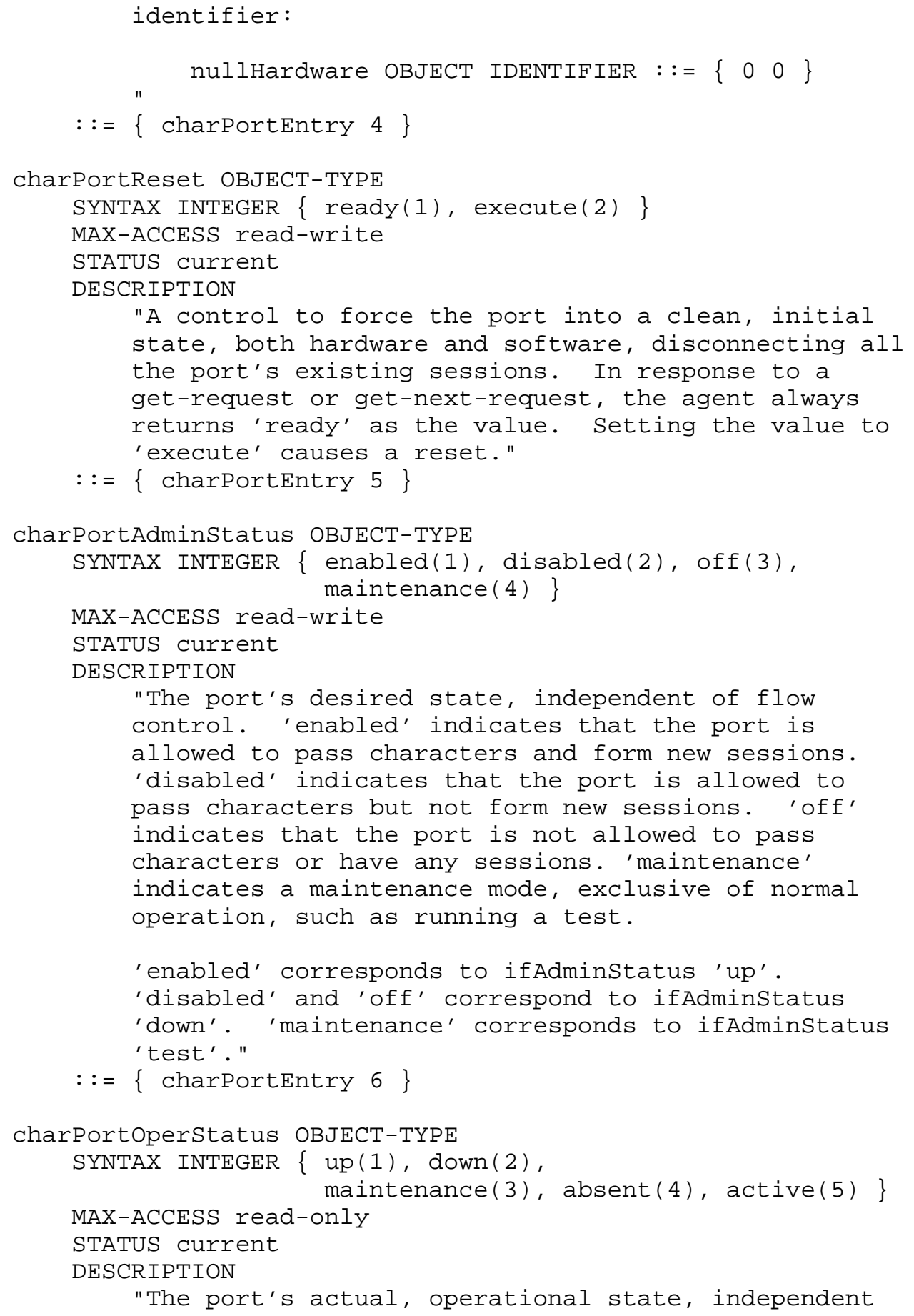




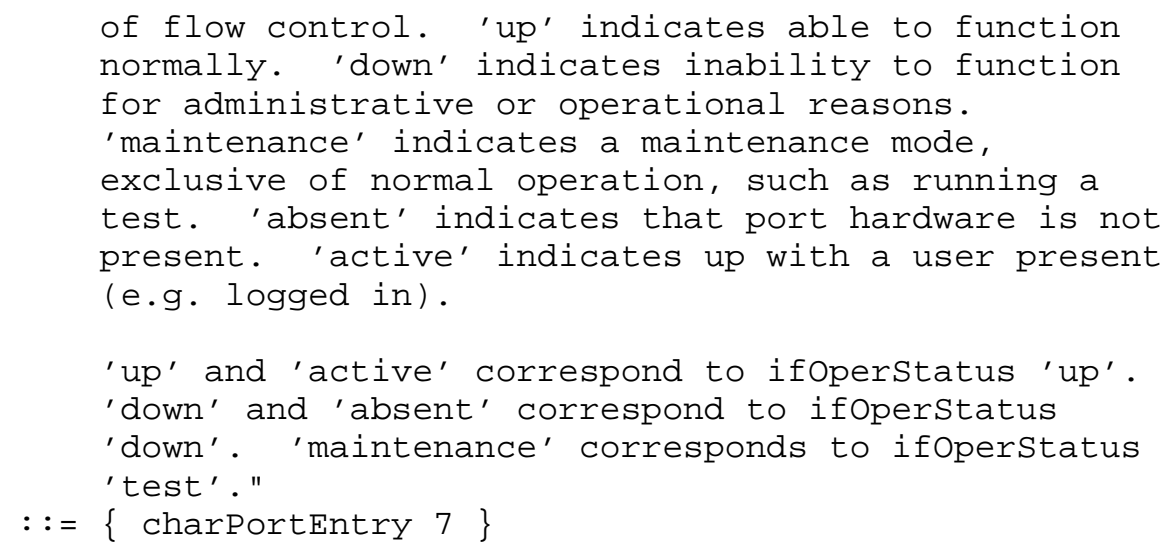

charPortlastchange OBJECT-TYPE

SYNTAX TimeTicks

MAX-ACCESS read-only

STATUS current

DESCRIPTION

"The value of sysupTime at the time the port entered its current operational state. If the current state was entered prior to the last reinitialization of the local network management subsystem, then this object contains a zero value."

$::=\{$ charportEntry 8$\}$

-- charPortInFlowType is deprecated in favor of

-- charPortInFlowTypes

charPortInFlowType OBJECT-TYPE

SYNTAX INTEGER \{ none(1), xonXoff(2), hardware(3),

MAX-ACCESS read-write ctsRts (4), dsrDtr(5) \}

STATUS deprecated

DESCRIPTION

"The port's type of input flow control. 'none' indicates no flow control at this level or below. 'xonXoff' indicates software flow control by recognizing XON and XOFF characters. 'hardware' indicates flow control delegated to the lower level, for example a parallel port.

'ctsRts' and 'dsrDtr' are specific to RS-232-like ports. Although not architecturally pure, they are included here for simplicity's sake."

$::=\{$ charportEntry 9$\}$ 


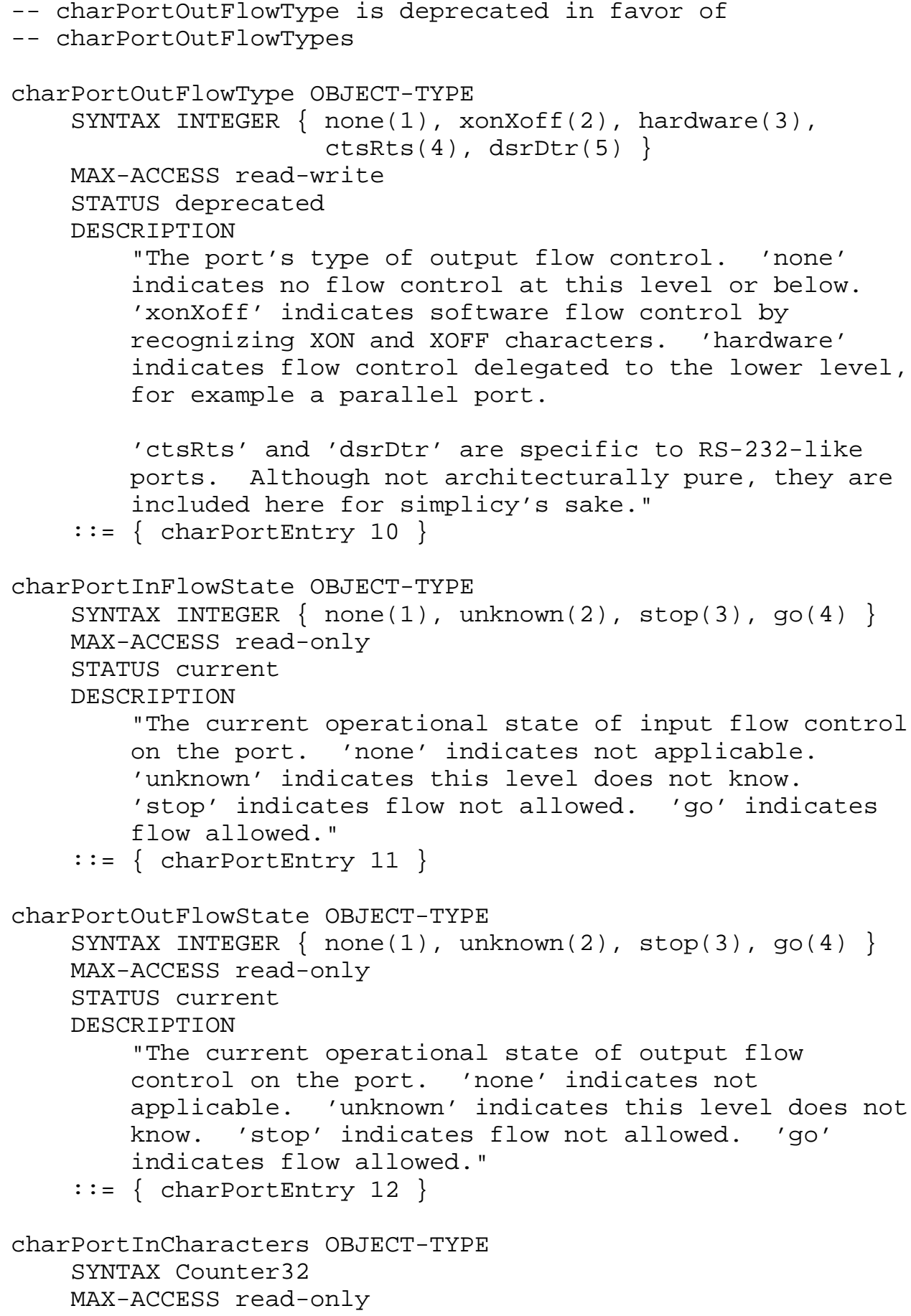




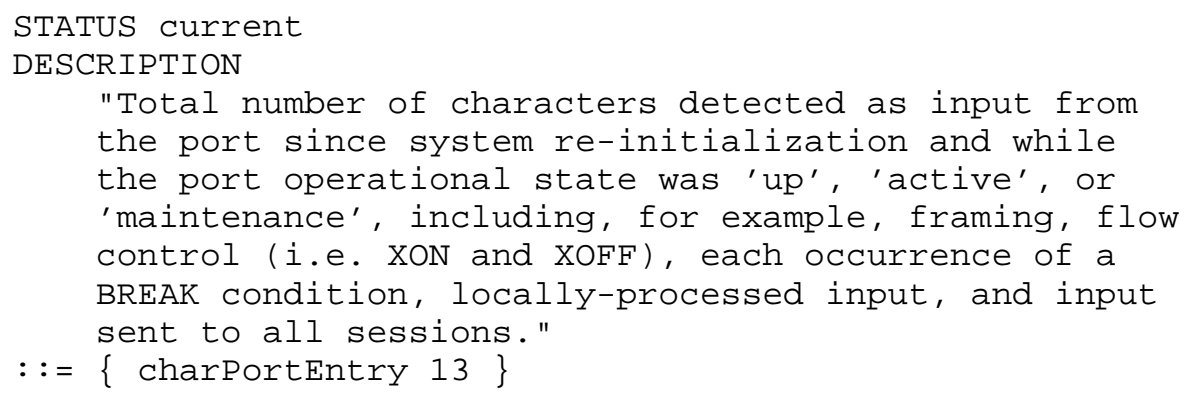




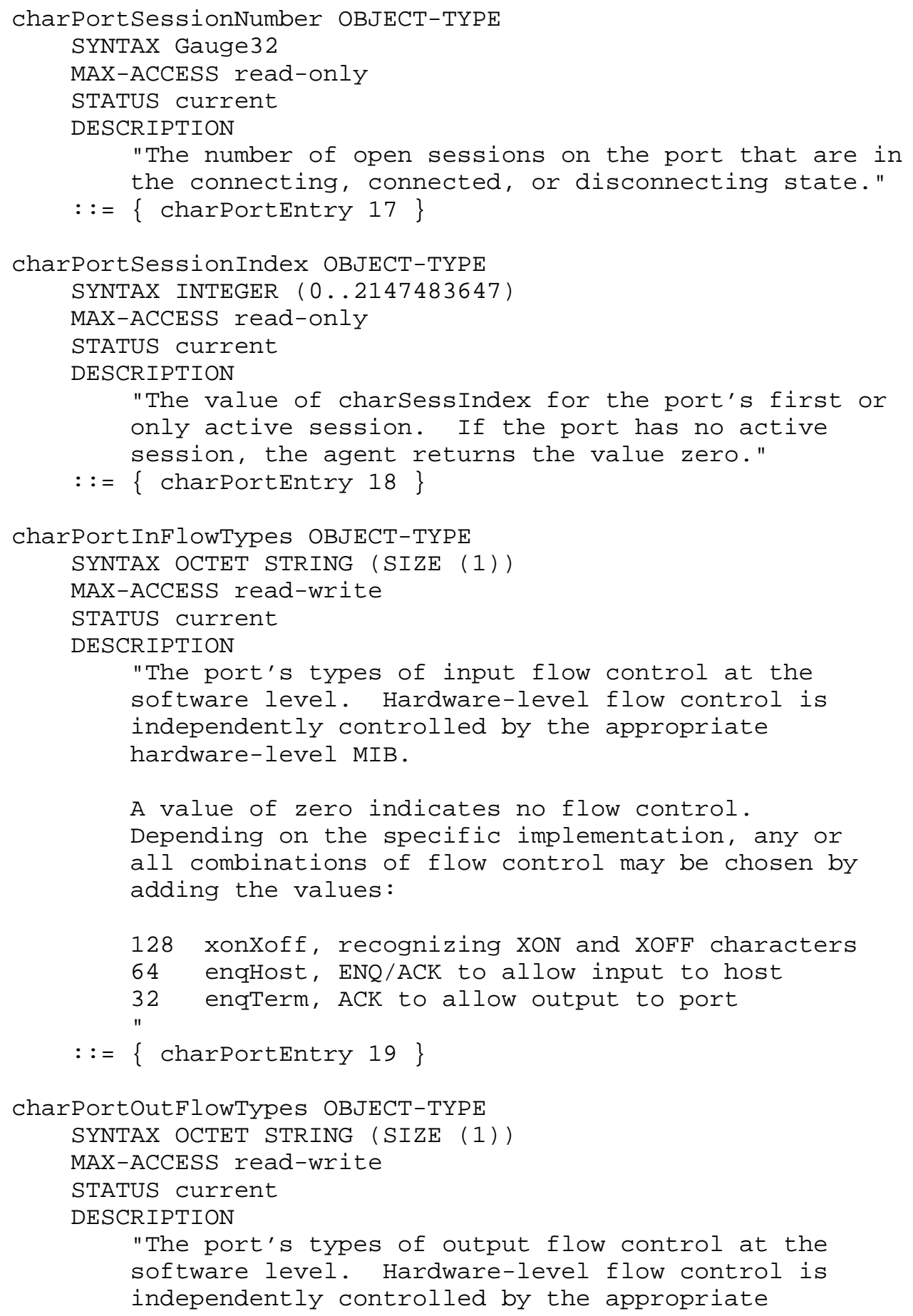




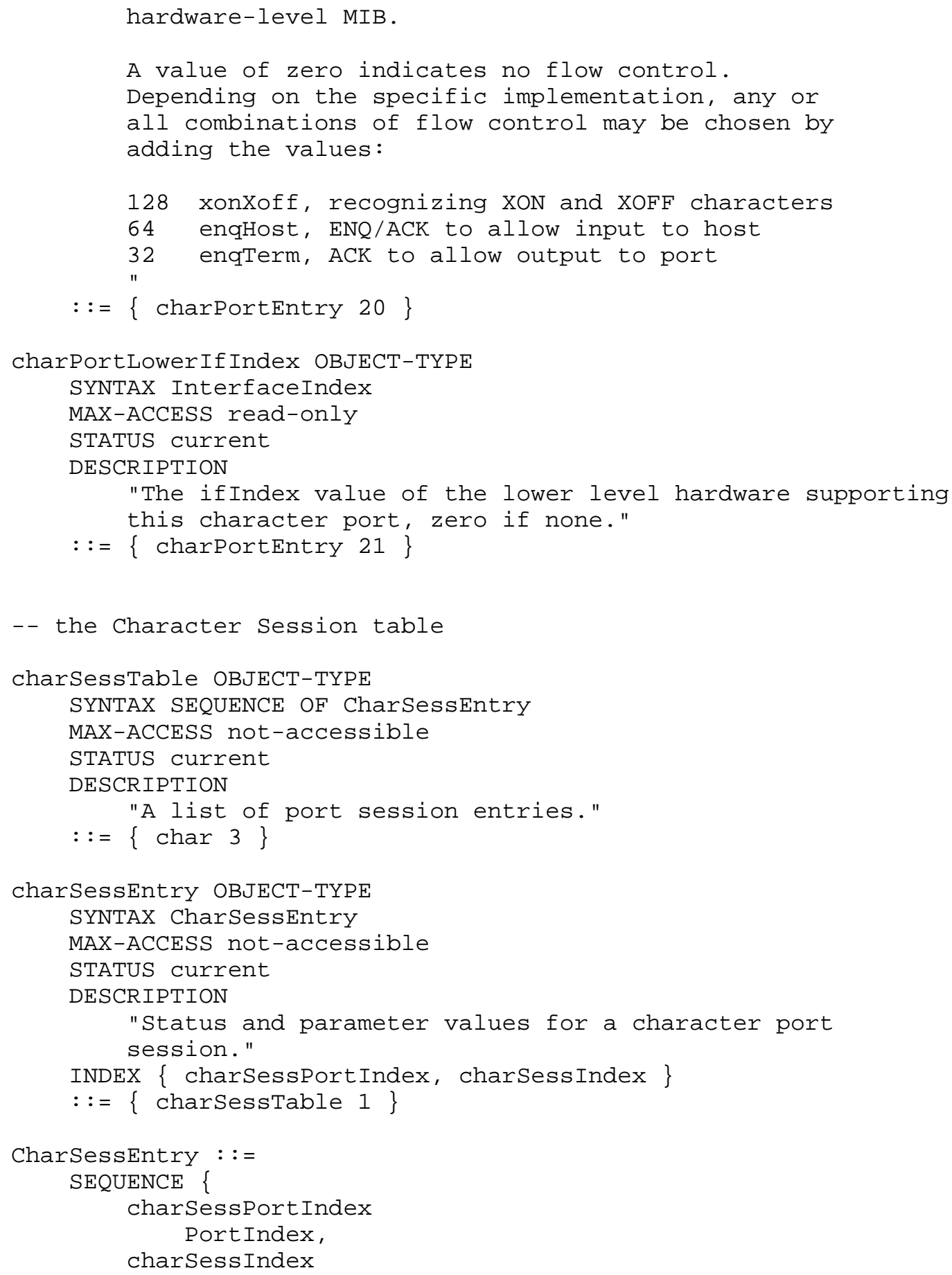




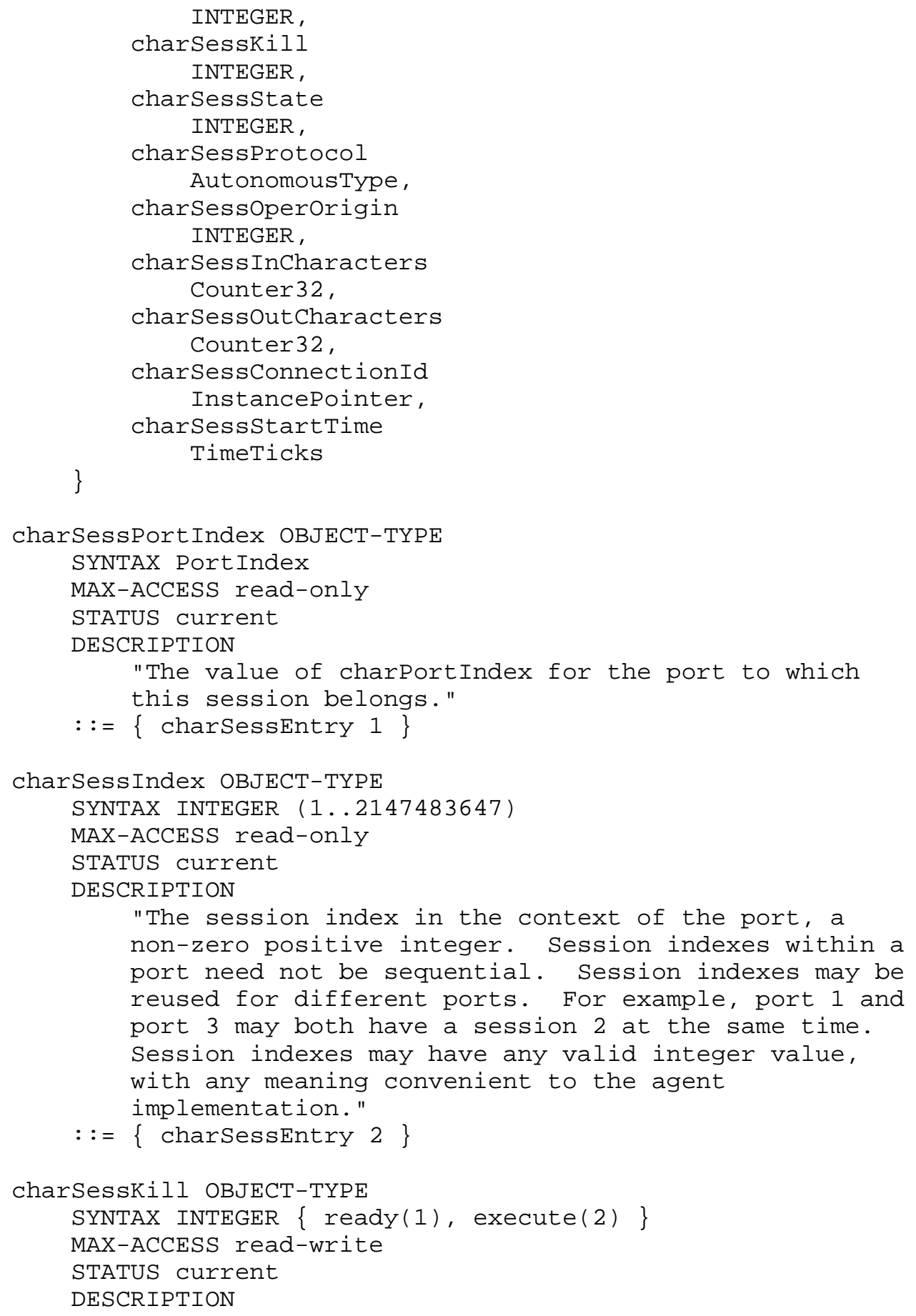




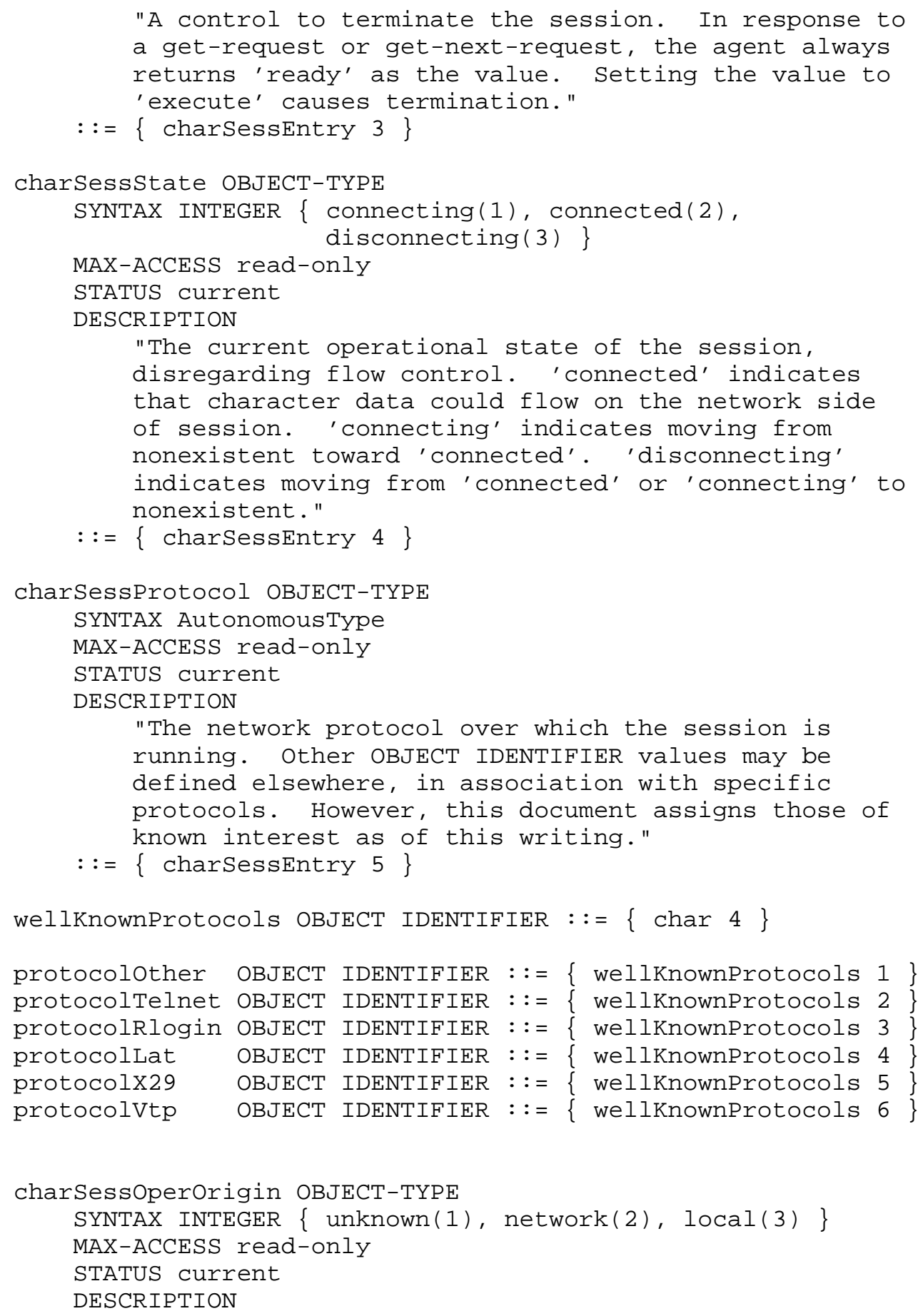




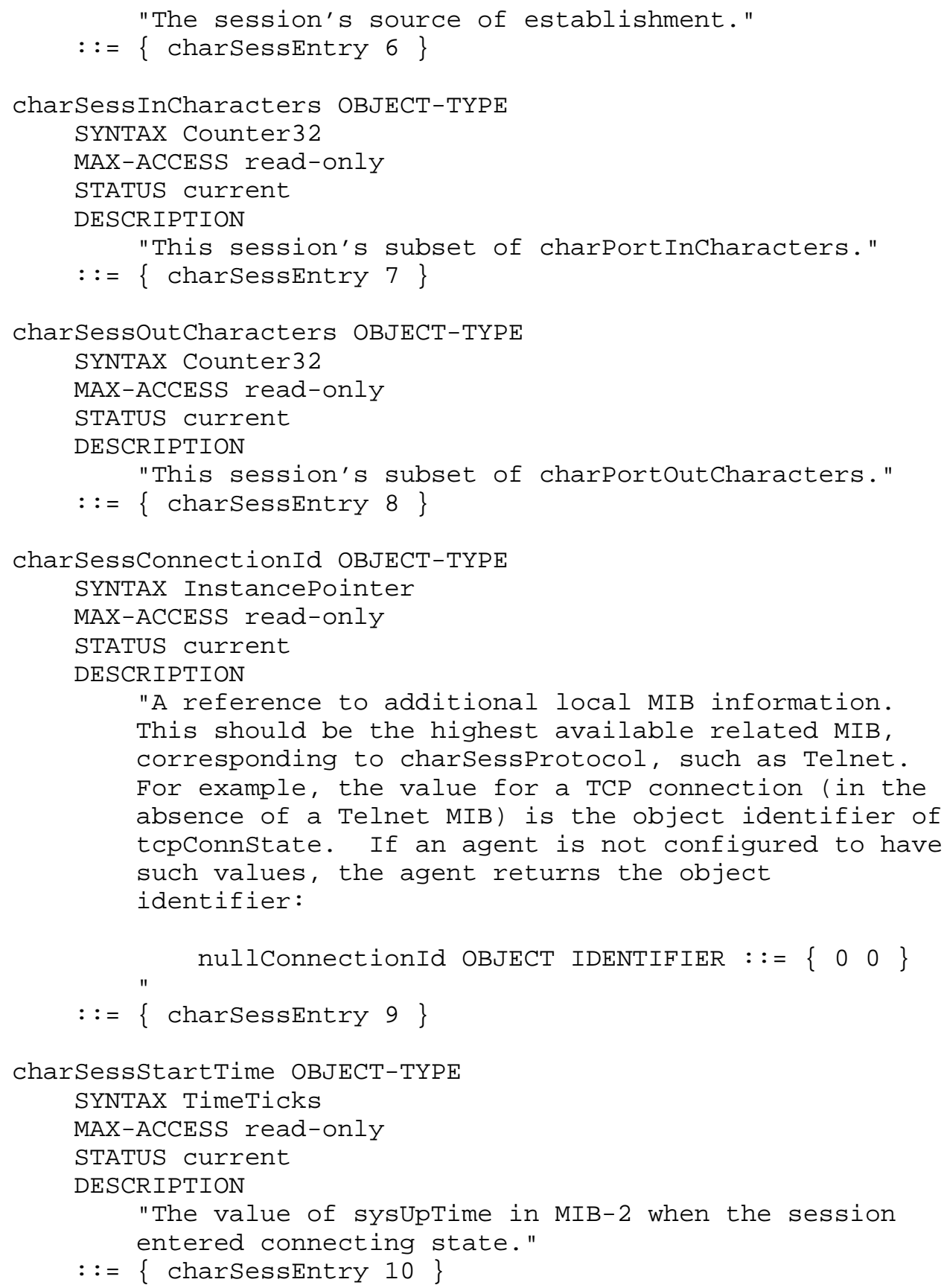


-- conformance information

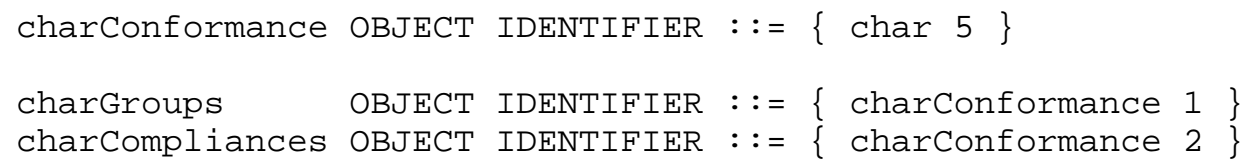

"A collection of objects providing information applicable to all Character interfaces."

END 


\section{Acknowledgements}

This memo was produced by the IETF Character MIB Working Group.

6. References

[1] Case, J., McCloghrie, K., Rose, M., and S. Waldbusser, "Structure of Management Information for version 2 of the Simple Network Management Protocol (SNMPv2)", RFC 1442, SNMP Research, Inc., Hughes LAN Systems, Dover Beach Consulting, Inc., Carnegie Mellon University, April 1993.

[2] McCloghrie, K., and M. Rose, Editors, "Management Information Base for Network Management of TCP/IP-based internets: MIB-II", STD 17, RFC 1213, Hughes LAN Systems, Performance systems International, March 1991.

[3] Galvin, J., and K. McCloghrie, "Administrative Model for version 2 of the Simple Network Management Protocol (SNMPv2)", RFC 1445, Trusted Information Systems, Hughes LAN Systems, April 1993.

[4] Case, J., McCloghrie, K., Rose, M., and S. Waldbusser, "Protocol Operations for version 2 of the Simple Network Management Protocol (SNMPv2)", RFC 1448, SNMP Research, Inc., Hughes LAN Systems, Dover Beach Consulting, Inc., Carnegie Mellon University, April 1993.

[5] McCloghrie, K., and F. Kastenholz, "Evolution of the Interfaces Group of MIB-II", RFC 1573, Hughes LAN Systems, FTP Software, January 1994 .

[6] Stewart, B., "Definitions of Managed Objects for RS-232-like Hardware Devices using SMIv2", RFC 1659, Xyplex, Inc., July 1994.

[7] Stewart, B., "Definitions of Managed Objects for Parallelprinter-like Hardware Devices using SMIv2", RFC 1660, Xyplex, Inc., July 1994. 
7. Security Considerations

Security issues are not discussed in this memo.

8. Author's Address

Bob Stewart

Xyplex, Inc.

295 Foster street

Littleton, MA 01460

Phone: 508-952-4816

Fax: 508-952-4887

EMail: rlstewart@eng.xyplex.com 